\title{
Regularity for a Class of Elliptic Operators with Dini Continuous Coefficients
}

\author{
Michael Taylor*
}

\begin{abstract}
We obtain regularity results for solutions to $P u=f$ when $P$ is a $k$ th order elliptic differential operator with the property that both $P$ and $P^{t}$ have coefficients that are continuous, with a modulus of continuity satisfying a Dini-type condition. Operators of this form arise in the study of regularity of functions that are known to be regular along the leaves of several foliations, such as arise in Anosov systems. The results here complement some previous results of the author and J. Rauch.
\end{abstract}

\section{Introduction}

In [11] the following elliptic regularity result was established.

Theorem 1.1 Let $P$ be an elliptic differential operator of order $k$ on an open set $\mathcal{O} \subset \mathbb{R}^{n}$ (perhaps an $N \times N$ system). Assume

$$
P \text { and } P^{t} \text { have coefficients in } L^{\infty} \cap \text { vmo. }
$$

Let $1<q \leq p<\infty$. Then

$$
u \in L_{\mathrm{loc}}^{q}(\mathcal{O}), P u \in L_{\mathrm{loc}}^{p}(\mathcal{O}) \Longrightarrow u \in H_{\mathrm{loc}}^{k, p}(\mathcal{O}) .
$$

By ellipticity here, we mean the following. The principal symbol of $P, P_{k}(x, \xi)$, is an $N \times N$ matrix-valued function, which is a polynomial in $\xi$, homogeneous of degree $k$. To say that $P$ is elliptic is to say that $P_{k}(x, \xi)$ is invertible whenever $\xi \neq 0$, and

$$
\left\|P_{k}(x, \xi)^{-1}\right\| \leq C|\xi|^{-k} .
$$

${ }^{*}$ Key words. elliptic equation, regularity, Sobolev spaces, Besov spaces, pseudodifferential operator, foliations

2000 Math Subject Classification: Primary 35J45, 35S05 
The work in [11] was motivated by the appearance in [10] and [9] of elliptic operators that were produced to apply to a function $u$ that was assumed to be smooth of order $k$ along the leaves of $N$ foliations $\mathcal{F}_{j}$ of $\mathcal{O}$. The ellipticity arises when, for each $x \in \mathcal{O}$,

$$
\mathbb{R}^{n}=\operatorname{Span}\left\{T_{x} \mathcal{F}_{j}: 1 \leq j \leq N\right\} .
$$

Such a family of foliations arises, for example, as the pair of stable and unstable foliations of a $C^{k+2}$ Anosov map. Typically, the leaves of such foliations are individually smooth, but they vary in a much less smooth fashion. The elliptic operators $P$ arising in these last two papers are scalar, have continuous coefficients and even order, and satisfy $P^{t}=P$. For the reader's convenience, and also to help motivate the study of this somewhat unusual class of elliptic operators, we briefly sketch how such foliations give rise to such operators $P$, referring to [10], [9], and [11] for more details.

Each foliation $\mathcal{F}_{j}$ has the property that there is a homeomorphism

$$
\Gamma_{j}: U_{j} \times V_{j} \longrightarrow \mathcal{O}_{j} \supset \mathcal{O}
$$

where $U_{j} \subset \mathbb{R}^{n-m_{j}}, V_{j} \subset \mathbb{R}^{m_{j}}$ are open sets, such that for each $x \in U_{j},\left\{\Gamma_{j}(x, y): y \in V_{j}\right\}$ is a leaf in $\mathcal{F}_{j}$. As for regularity of $\mathcal{F}_{j}$, we fix $r \in[0, \infty)$ and $k \in \mathbb{N}$, and assume

$$
\partial_{y}^{\alpha} \Gamma_{j} \in C^{r}\left(U_{j} \times V_{j}\right), \quad \forall|\alpha| \leq k .
$$

Here $C^{0}\left(U_{j} \times V_{j}\right)$ denotes the space of continuous functions on $U_{j} \times V_{j}$. If $r=\ell+\alpha, \ell \in$ $\mathbb{Z}^{+}, 0<\alpha<1, C^{r}\left(U_{j} \times V_{j}\right)$ denotes the space of functions with derivatives of order $\leq \ell$ Hölder continuous of exponent $\alpha$. We also assume that for each fixed $x$, the map $y \mapsto \Gamma_{j}(x, y)$ is a $C^{k}$ immersion of $V_{j}$ into $\mathcal{O}_{j}$, and that $\Gamma_{j}$ is absolutely continuous in the sense that for Borel sets $E \subset U_{j}, F \subset V_{j}$, the measure of $\Gamma_{j}(E \times F) \subset \mathcal{O}_{j}$ is given by

$$
\int_{E} \int_{F} J_{j}(x, y) d y d x,
$$

and we assume $J_{j}>0$ on $U_{j} \times V_{j}$ and

$$
\partial_{y}^{\alpha} J_{j} \in C^{r}\left(U_{j} \times V_{j}\right), \quad \forall|\alpha| \leq k .
$$

If these conditions hold, we say $\mathcal{F}_{j}$ is regular of order $(k, r)$. The papers [10], [9], and [11] paid particular attention to cases where $r=0$.

Given a function $u$ on $\mathcal{O}_{j}$, we say

$$
u \in H_{\mathcal{F}_{j}}^{k, p}\left(\mathcal{O}_{j}\right) \Longleftrightarrow \partial_{y}^{\alpha}\left(u \circ \Gamma_{j}\right) \in L^{p}\left(U_{j} \times V_{j}\right), \quad \forall|\alpha| \leq k .
$$


A basic problem had been to establish regularity for $u$ on $\mathcal{O}$, given that $u$ satisfies (1.9) for each $j$, and given the spanning hypothesis (1.4). An approach, suggested in [9], proceeds as follows. Take $k=2 \ell$. We define the operator $P_{j}$ by

$$
\left(P_{j} u\right) \circ \Gamma_{j}=\sum_{|\beta|=\ell}\left(-\partial_{y}\right)^{\beta}\left(\partial_{y}^{\beta}\right)\left(J_{j} u \circ \Gamma_{j}\right)
$$

for each $j$ a differential operator of order $2 \ell=k$. Then set

$$
P=\sum_{j} P_{j}
$$

The hypothesis (1.4) implies that $P$ is an elliptic differential operator on $\mathcal{O}$, of order $k$. The hypothesis that each $\mathcal{F}_{j}$ is regular of order $(k, 0)$ implies $P$ has continuous coefficients, i.e.,

$$
P=\sum_{|\alpha| \leq k} a_{\alpha}(x) \partial^{\alpha}, \quad a_{\alpha} \in C^{0}(\mathcal{O})
$$

Furthermore, we have (with $z=\Gamma(x, y)$ )

$$
\begin{aligned}
\int u\left(\overline{P_{j} v}\right) d z & =\sum_{|\beta|=\ell} \int \partial_{y}^{\beta}\left(J_{j} u \circ \Gamma_{j}\right) \partial_{y}^{\beta}\left(J_{j} \overline{v \circ \Gamma_{j}}\right) d x d y \\
& =\int\left(P_{j} u\right) \bar{v} d z
\end{aligned}
$$

so

$$
P=P^{t}
$$

If (1.9) holds for all $j$, we have

$$
u, P u \in L^{p}(\mathcal{O}) \text {. }
$$

In [11], the authors were challenged to establish the regularity result (1.2), which apparently did not follow from such classical treatments as in [1]. With the techniques developed in [11] to establish this regularity result, the additional generality for the class of operators treated in Theorem 1.1 arose naturally, the passage from continuous coefficients to $L^{\infty} \cap$ vmo facilitated by work of [4]. There are several motivations to establish Theorem 1.1 in the setting of $N \times N$ systems, rather than simply the scalar case. For one, the techniques work in this more general setting. In fact, as the reader will see in the arguments given here, even if we start with a scalar equation, we produce systems. Another reason to be interested in the systems case is that we can get results, not just for real-valued functions smooth along foliations, in the sense of (1.9), but also for sections of a smooth vector bundle, with estimates on $k$-fold covariant derivatives along the leaves of such foliations. 
So far, we have emphasized foliations regular of order $(k, 0)$. However, the foliation setup also allows for such operators $P$ with mildly regular coefficients. Such mild regularity allows the possibility to establish some endpoint cases extending (1.2). In this connection, it was also shown in [11] that if to (1.1) one adds the hypothesis

$$
P^{t} \text { has Hölder continuous coefficients, }
$$

then

$$
u \in \mathcal{M}(\mathcal{O}), P u \in L_{\mathrm{loc}}^{p}(\mathcal{O}) \Longrightarrow u \in H_{\mathrm{loc}}^{k, p}(\mathcal{O})
$$

and if to (1.1) one adds the hypothesis

$$
P \text { has Hölder continuous coefficients, }
$$

then

$$
u \in L_{\mathrm{loc}}^{p}(\mathcal{O}), P u \in \operatorname{bmo}(\mathcal{O}) \Longrightarrow u \in \mathfrak{h}_{\text {loc }}^{k, \infty}(\mathcal{O}) .
$$

Here $\mathcal{M}(\mathcal{O})$ denotes the space of locally finite Borel measures on $\mathcal{O}$, bmo the localized John-Nirenberg space, and

$$
\mathfrak{h}^{k, \infty}(\mathcal{O})=\left\{u \in \operatorname{bmo}(\mathcal{O}): \partial^{\alpha} u \in \operatorname{bmo}(\mathcal{O}),|\alpha| \leq k\right\} .
$$

As usual $H^{k, p}$ denotes an $L^{p}$-Sobolev space. The localized space bmo was introduced in [6], making use of results on BMO in [5]. The space vmo in (1.1) is the corresponding localization of D. Sarason's subspace VMO $\subset$ BMO.

In this paper we want to prove such regularity results as (1.17) and (1.19), and others, under Dini-type hypotheses on the coefficients of $P$ and $P^{t}$. Such a situation would arise in the foliation context if (1.6) and (1.8) were replaced by inclusion in spaces with Dini-type modulus of continuity, for example. The study of elliptic operators with Dini continuous coefficients has a rich history, starting with classical constructions of isothermal coordinates on $2 \mathrm{D}$ surfaces with Dini continuous metric tensor (by contrast, [7] presents a $C^{0}$ metric tensor for which there are no $C^{1}$ isothermal coordinates; see also [8]). The necessity for having a Dini-type condition to obtain the implication (1.19) can be traced to the fact that bmo is not a module over $C^{0}$, but it is a module over the ring of Dini continuous functions (cf. [13], Chapter 1, (2.63)).

We work with spaces $C^{(\lambda)}$, introduced in Chapter I of [13], defined by

$$
u \in C^{(\lambda)} \Longleftrightarrow\left\|\psi_{j}(D) u\right\|_{L^{\infty}} \leq C \lambda(j) .
$$

Here $\left\{\psi_{j}: j \in \mathbb{Z}^{+}\right\}$is a Littlewood-Paley partition of unity, with $\psi_{j}(\xi)$ supported on $|\xi| \sim 2^{j}$. We will take

$$
\lambda(j)=\langle j\rangle^{-s}
$$


where $\langle x\rangle=\left(2+|x|^{2}\right)^{1 / 2}$. As long as $s>1$, an element $u \in C^{(\lambda)}$ will be continuous, and satisfy

$$
|u(x+y)-u(x)| \leq C \omega(|y|)
$$

with

$$
\omega(h)=\left(\log \frac{1}{h}\right)^{-s+1},
$$

for $0<h \leq 1 / e$. On the other hand, a function $u$ satisfying $(1.23)$ with $\omega(h)=(\log 1 / h)^{-s}$ will belong to $C^{(\lambda)}$ with $\lambda(j)$ given by (1.22). Otherwise said, with

$$
\sigma(h)=\left(\log \frac{1}{h}\right)^{-s}
$$

for $0<h \leq 1 / e$, we have

$$
C^{\sigma} \subset C^{(\lambda)} \subset C^{\omega}
$$

as shown in (1.30) in Chapter I of [13]. We recall that a function is said to be Dini continuous if it has a modulus of continuity $\beta(h)$ satisfying

$$
\int_{0}^{1} \frac{\beta(h)}{h} d h<\infty
$$

Note that $\omega(h)$, given by (1.24), has this property if and only if $s>2$.

One of our goals is to establish the following.

Theorem 1.2 In the setting of Theorem 1.1, assume

$$
P \text { and } P^{t} \text { have coefficients in } C^{(\lambda)}, \quad \lambda(j)=\langle j\rangle^{-s}, \quad s>1 .
$$

Then the implication (1.17) holds. If in addition P has Dini continuous coefficients, then (1.19) holds.

A key ingredient in the proof of Theorem 1.2 is the following result, of independent interest, involving regularity in Besov spaces $B_{p, q}^{s}$.

Theorem 1.3 Let $P$ be an elliptic differential operator of order $k$ on $\mathcal{O}$, and assume (1.28) holds. Take $p, q, p_{1}, q_{1} \in[1, \infty]$. Then

$$
u \in B_{p_{1}, q_{1}(\mathrm{loc})}^{0}(\mathcal{O}), P u \in B_{p, q(\mathrm{loc})}^{0}(\mathcal{O}) \Longrightarrow u \in B_{p, q(\mathrm{loc})}^{k}(\mathcal{O}) .
$$

More generally, for $1 \leq j \leq k$,

$$
u \in B_{p_{1}, q_{1}(\mathrm{loc})}^{0}(\mathcal{O}), P u \in B_{p, q \text { (loc })}^{-k+j}(\mathcal{O}) \Longrightarrow u \in B_{p, q(\mathrm{loc})}^{j}(\mathcal{O}) .
$$


The applicability of Besov space estimates to the proof of Theorem 1.2 arises from the inclusions

$$
B_{1,1}^{0} \subset L^{1} \subset \mathcal{M} \subset B_{1, \infty}^{0}, \quad B_{\infty, 1}^{0} \subset L^{\infty} \subset \text { bmo } \subset B_{\infty, \infty}^{0} .
$$

For example, if (1.28) holds, we can apply (1.29) with $p_{1}=1, q_{1}=\infty, p=p, q=\infty$, to get

$$
u \in \mathcal{M} \subset B_{1, \infty \text { (loc })}^{0}, P u \in L_{\mathrm{loc}}^{p} \subset B_{p, \infty(\mathrm{loc})}^{0} \Rightarrow u \in B_{p, \infty(\mathrm{loc})}^{k} \subset L_{\mathrm{loc}}^{p},
$$

the last inclusion holding whenever $k \geq 1$. From here, $(1.2)$ applies to give $u \in H_{\text {loc }}^{k, p}(\mathcal{O})$, yielding the implication (1.17). As for the implication (1.19), we can apply (1.29) with $p_{1}=p, q_{1}=\infty, p$ replaced by $\infty$, and $q=\infty$, to get

$$
u \in L_{\mathrm{loc}}^{p} \subset B_{p, \infty \text { (loc) }}^{0}, P u \in \operatorname{bmo} \subset B_{\infty, \infty \text { (loc) }}^{0} \Rightarrow u \in B_{\infty, \infty \text { (loc) }}^{k} .
$$

Thus the proof of Theorem 1.2 is finished with the following result.

Theorem 1.4 Let $P$ be an elliptic differential operator of order $k$ on $\mathcal{O}$, and assume

$$
P \text { has coefficients in } C^{(\lambda)}, \quad \lambda(j)=\langle j\rangle^{-s}, \quad s>1,
$$

and also that P has Dini continuous coefficients. Then

$$
u \in B_{\infty, \infty \text { (loc) }}^{k}(\mathcal{O}), P u \in \operatorname{bmo}(\mathcal{O}) \Longrightarrow u \in \mathfrak{h}_{\text {loc }}^{k, \infty}(\mathcal{O}) .
$$

We are left with the task of proving Theorems 1.3-1.4. Theorem 1.4 is proven via a parametrix construction. More precisely, we split $P$ into two pieces, $P=P^{\#}+P^{b}$, where $P^{\#}$ has a parametrix $E^{\#}$ that is smoothing of order $k$ on a wide variety of function spaces, while $E^{\#} P^{b}$ maps certain strategically chosen function spaces to spaces with ever so slightly greater regularity. This proof will be done in $\S 4$.

The proof of Theorem 1.3 involves two steps, as did the proof of Theorem 1.1 given in [11]. The first step is a globalization argument, in which we produce an elliptic operator $L$ on the torus $\mathbb{T}^{n}$ which is shown to be invertible from $B_{p, q}^{s+k}\left(\mathbb{T}^{n}\right)$ to $B_{p, q}^{s}\left(\mathbb{T}^{n}\right)$, for $p, q \in[1, \infty]$ and $-k \leq s \leq 0$. The second step is a localization argument, obtaining results of the form (1.29) and (1.30) from the corresponding global results for $L$, via cutoffs and commutator estimates.

In order to obtain such $L$, we make some preliminary transformations of $P$. Clearly the local regularity results (1.29) and (1.30) hold for $P$ if they hold for the $2 N \times 2 N$ system

$$
P_{2}=\left(\begin{array}{cc}
0 & P^{t} \\
P & 0
\end{array}\right)
$$

We begin to construct an operator on the torus. Without loss of generality, we can assume $\mathcal{O} \subset \mathbb{R}^{n}$ is bounded. Put this set in a box and identify opposite edges, to regard $\mathcal{O} \subset \mathbb{T}^{n}$. 
If we want to establish local regularity on a neighborhood $\Omega$ of $x_{0} \in \mathcal{O}$, pick $\varphi \in C_{0}^{\infty}(\mathcal{O})$, equal to 1 on $\bar{\Omega}$, satisfying $0 \leq \varphi \leq 1$, set $\psi=1-\varphi$, and form

$$
L_{2} u=\varphi P_{2}(\varphi u)+i \psi(-\Delta)^{k / 2}(\psi u),
$$

where $u$ takes values in $\mathbb{C}^{2 N}$ and $(-\Delta)^{k / 2}$ acts on $\psi u$ componentwise. This is a differential operator if $k$ is even, and otherwise not, but in any case, for a variety of function spaces $X$,

$$
u \in X\left(\mathbb{T}^{n}\right) \Longrightarrow L_{2} u=P_{2} u \text { on } \Omega .
$$

Hence, to obtain the desired local regularity (1.29) and (1.30), on $\Omega$, it suffices to establish

$$
u \in B_{p_{1}, q_{1}(\mathrm{loc})}^{0}(\Omega), L_{2} u \in B_{p, q(\mathrm{loc})}^{-k+j}(\Omega) \Longrightarrow u \in B_{p, q(\mathrm{loc})}^{j}(\Omega)
$$

for $1 \leq j \leq k, p, q, p_{1}, q_{1} \in[1, \infty]$. The operator $L_{2}$ is a pseudodifferential operator with rough symbol; more precisely,

$$
L_{2} \in O P C^{(\lambda)} S_{\mathrm{cl}}^{k}\left(\mathbb{T}^{n}\right) .
$$

The symbol class $C^{(\lambda)} S_{\mathrm{cl}}^{k}$, introduced in Chapter I of [13], is defined in Appendix A of this paper. Furthermore, since $P_{2}=P_{2}^{t}$ and $(-\Delta)^{k / 2}$ is scalar, it is readily established that $L_{2}$ is elliptic. In addition, it is important (and easily seen) that we have

$$
L_{2}^{t} \in O P C^{(\lambda)} S_{\mathrm{cl}}^{k}\left(\mathbb{T}^{n}\right) .
$$

We again desire formal self-adjointness, so we form the $4 N \times 4 N$ system

$$
L_{1}=\left(\begin{array}{cc}
0 & L_{2}^{t} \\
L_{2} & 0
\end{array}\right)
$$

and note that it suffices to establish the result (1.39) with $L_{2}$ replaced by $L_{1}$, which is also elliptic in $O P C^{(\lambda)} S_{\mathrm{cl}}^{k}\left(\mathbb{T}^{n}\right)$.

The operator $L$ will be of the form $L=L_{1}-\lambda I$, with appropriately chosen $\lambda \in \mathbb{R}$. This construction will be made in $\S 2$, where we proceed from Fredholm results on $L_{1}$ to invertibility results on $L$. From here we derive the proof of Theorem 1.3 in $\S 3$. In $\S 4$ we prove Theorem 1.4 and also a related result, namely that under the hypotheses of (1.28),

$$
u \in B_{1, \infty \text { (loc) }}^{0}(\mathcal{O}), P u \in B_{1,1 \text { (loc) }}^{-k}(\mathcal{O}) \Longrightarrow u \in B_{1,1 \text { (loc) }}^{0}(\mathcal{O}),
$$

which is needed in the proof of one case of Proposition 2.2.

Our arguments require some results on boundedness of rough pseudodifferential operators on $B_{p, q}^{0}$, which we establish in Appendix B, following Appendix A, where we recall the definitions of several classes of rough pseudodifferential operators from [13], including classes like (1.40), and other classes, obtained from (1.40) by the process of "symbol smoothing." 


\section{Global results}

We begin with some Fredholm results on the operator $L_{1}$ constructed in $\S 1$. More generally, we take

$$
L_{1} \in O P C^{(\lambda)} S_{\mathrm{cl}}^{k}\left(\mathbb{T}^{n}\right), \quad \text { elliptic, } \quad L_{1}=L_{1}^{t}
$$

Here we assume

$$
\lambda(j)=\langle j\rangle^{-s}, \quad s>1 .
$$

Here, ellipticity of $L_{1}$ means the principal symbol of $L_{1}$ is invertible for $|\xi| \geq 1$ and satisfies an estimate parallel to (1.3).

Proposition 2.1 For such $L_{1}$, and with $p, q \in[1, \infty]$, we have

$$
L_{1}: B_{p, q}^{k}\left(\mathbb{T}^{n}\right) \longrightarrow B_{p, q}^{0}\left(\mathbb{T}^{n}\right)
$$

and

$$
L_{1}: B_{p, q}^{0}\left(\mathbb{T}^{n}\right) \longrightarrow B_{p, q}^{-k}\left(\mathbb{T}^{n}\right)
$$

Fredholm, of index 0 .

Proof. First take $A=L_{1}(1-\Delta)^{-k / 2}$, elliptic in $O P C^{(\lambda)} S_{\mathrm{cl}}^{0}$. The Fredholm property in (2.3) is equivalent to the statement that

$$
A: B_{p, q}^{0}\left(\mathbb{T}^{n}\right) \longrightarrow B_{p, q}^{0}\left(\mathbb{T}^{n}\right)
$$

is Fredholm. To show this, we use symbol smoothing, writing

$$
A=A^{\#}+A^{b}, \quad A^{\#} \in O P S_{1, \delta}^{0}, \text { elliptic, }
$$

where $\delta$ is chosen in $(0,1)$, and

$$
A^{b} \in O P C^{(\lambda)} S_{1,(\tau)}^{-\psi}
$$

where, upon choosing $\gamma \in(0, s-1)$, we can set

$$
\langle\xi\rangle^{-\psi(\xi)}=\left(\log _{2}\langle\xi\rangle\right)^{-(s-1-\gamma)}, \quad \tau(\xi)=\left(\log _{2}\langle\xi\rangle\right)^{s-1-\gamma} .
$$

Cf. [13], Chapter I, §3, especially Proposition 3.2 and (3.28)-(3.32). The definition of the symbol class in (2.7) is recalled in Appendix A. Mapping properties on Besov spaces given in [13], Chapter I, $\S 12$ (cf. also Appendix B of this paper) show that $A^{\#}$ is Fredholm on $B_{p, q}^{0}\left(\mathbb{T}^{n}\right)$, so it remains to show that

$$
A^{b} \text { is compact on } B_{p, q}^{0}\left(\mathbb{T}^{n}\right) \text {, }
$$


to establish that $A$ is Fredholm in (2.5). In fact, Proposition B.1 gives

$$
O P C^{(\lambda)} S_{1,(\tau)}^{0}: B_{p, q}^{0}\left(\mathbb{T}^{n}\right) \longrightarrow B_{p, q}^{0}\left(\mathbb{T}^{n}\right),
$$

when (2.2) and (2.8) hold and $0<\gamma<s-1$, so it remains to show that, for

$$
\begin{aligned}
& q(\xi)=\left(\log _{2}\langle\xi\rangle\right)^{-(s-1-\gamma)}, \quad 0<s-1-\gamma, \\
& q(D): B_{p, q}^{0}\left(\mathbb{T}^{n}\right) \longrightarrow B_{p, q}^{0}\left(\mathbb{T}^{n}\right) \text { is compact. }
\end{aligned}
$$

To see this, take $\varphi \in C_{0}^{\infty}\left(\mathbb{R}^{n}\right)$, equal to 1 on a neighborhood of 0 , and consider

$$
q_{\varepsilon}(\xi)=\varphi(\varepsilon \xi) q(\xi) .
$$

It is readily verified that, as $\varepsilon \rightarrow 0, q(\xi)-q_{\varepsilon}(\xi) \rightarrow 0$ in $S_{1,0}^{0}$, a Fréchet space with seminorms implicitly recorded in (A.6). Now elements of $O P S_{1,0}^{0}$ are bounded on the Besov spaces $B_{p, q}^{0}\left(\mathbb{T}^{n}\right)$ (this is a classical result, of which Proposition B.1 is a refinement). Hence $q_{\varepsilon}(D) \rightarrow$ $q(D)$ in operator norm on these Besov spaces. Since $q_{\varepsilon}(D)$ has finite rank for each $\varepsilon>0$, compactness of $q(D)$ follows.

Thus we have Fredholmness in (2.5) and hence in (2.3). The index 0 calculation in (2.3) then follows from the standard homotopy argument involving

$$
A_{\tau}=\tau A+(1-\tau) i I, \quad 0 \leq \tau \leq 1,
$$

which, we note, is elliptic in $O P C^{(\lambda)} S_{\mathrm{cl}}^{0}$ for each $\tau \in[0,1]$, since the principal symbol of $A$ is self-adjoint and elliptic and the principal symbol of the identity $I$ is scalar and elliptic.

The proof that $L_{1}$ is Fredholm of index 0 in (2.4) is similar. This time write $A=$ $(1-\Delta)^{-k / 2} L_{1} \in \varnothing P C^{(\lambda)} S_{\mathrm{cl}}^{0}$, elliptic. (See also Appendix A for definitions of this operator class and the ones appearing in (2.13)-(2.14).) We have a decomposition of the form (2.6), with

$$
A^{b} \in \varnothing P C^{(\lambda)} S_{1,(\tau)}^{-\psi},
$$

and (2.8) holding. Parallel to (2.10), we have

$$
\varnothing P C^{(\lambda)} S_{1,(\tau)}^{0}: B_{p, q}^{0}\left(\mathbb{T}^{n}\right) \longrightarrow B_{p, q}^{0}\left(\mathbb{T}^{n}\right) .
$$

This can be deduced from the result

$$
O P C^{(\lambda)} S_{1,(\tau)}^{0}: B_{p^{\prime}, q^{\prime}}^{0}\left(\mathbb{T}^{n}\right) \longrightarrow B_{p^{\prime}, q^{\prime}}^{0}\left(\mathbb{T}^{n}\right),
$$

and the duality (cf. [15], p. 178)

$$
B_{p, q}^{0}\left(\mathbb{T}^{n}\right)^{\prime}=B_{p^{\prime}, q^{\prime}}^{0}\left(\mathbb{T}^{n}\right), \quad 1 \leq p, q<\infty .
$$


In fact, (2.14) then follows directly if $p>1$ and $q>1$. If either $p=1$ or $q=1$ it follows from the duality together with the fact that $C^{\infty}\left(\mathbb{T}^{n}\right)$ is a dense linear subspace of $B_{p, q}^{0}\left(\mathbb{T}^{n}\right)$, with the sole exception of $B_{1, \infty}^{0}\left(\mathbb{T}^{n}\right)$, in which $B_{2, \infty}^{0}\left(\mathbb{T}^{n}\right)$ is dense. This establishes (2.14). The result (2.11) again applies to give (2.9) in this context. This yields Fredholmness in (2.4), and the same argument given above applies to the index calculation, so we have Proposition 2.1 .

To proceed further, we will make use of a result established in [11], Lemma 3.2, (even for less regular coefficients). Namely, there is a discrete set $\mathcal{C} \subset \mathbb{R}$ such that, if we take $\lambda \in \mathbb{R} \backslash \mathcal{C}$ and set

$$
L=L_{1}-\lambda I,
$$

then, for $p \in(1, \infty)$, we have isomorphisms (indicated by the " $\approx$ " atop the arrows)

$$
\begin{aligned}
L: H^{k, p}\left(\mathbb{T}^{n}\right) & \stackrel{\approx}{\longrightarrow} L^{p}\left(\mathbb{T}^{n}\right), \\
L: L^{p}\left(\mathbb{T}^{n}\right) & \stackrel{\approx}{\longrightarrow} H^{-k, p}\left(\mathbb{T}^{n}\right) .
\end{aligned}
$$

We will now prove the following.

Proposition 2.2 With $\lambda \in \mathbb{R} \backslash \mathcal{C}$ and $L$ as in (2.18), we have for $p, q \in[1, \infty]$ isomorphisms

$$
\begin{aligned}
& L: B_{p, q}^{k}\left(\mathbb{T}^{n}\right) \stackrel{\approx}{\longrightarrow} B_{p, q}^{0}\left(\mathbb{T}^{n}\right), \\
& L: B_{p, q}^{0}\left(\mathbb{T}^{n}\right) \stackrel{\approx}{\longrightarrow} B_{p, q}^{-k}\left(\mathbb{T}^{n}\right),
\end{aligned}
$$

Proof. In each case, $L$ is a compact perturbation of $L_{1}$, so we know that $L$ is Fredholm of index 0. By (2.18) we know that the null space of $L$ in $L^{p}\left(\mathbb{T}^{n}\right)$ is zero for each $p>1$. This shows that $L$ is injective in (2.19), hence an isomorphism in this case. The isomorphism in (2.20) follows from (2.19) and duality (since $L=L^{t}$ ), unless $p=1$ or $q=1$.

To treat $q=1$ in $(2.20)$, note that $B_{p, 1}^{0}\left(\mathbb{T}^{n}\right) \subset L^{p}\left(\mathbb{T}^{n}\right)$, so if $p>1$ injectivity in $(2.20)$ follows from (2.18). To treat the case $p=1$ in (2.20), note that the annihilator of the range of $L$ in this case coincides with the null space of $L$ in $B_{\infty, q^{\prime}}^{k}\left(\mathbb{T}^{n}\right)$, as long as $q<\infty$, and we have isomorphism then.

This leaves the case $(p, q)=(1, \infty)$ in $(2.20)$. For this case, we can use Proposition 4.1 to deduce that elements of the null space of $L$ in $(2.20)$ actually belong to $B_{1,1}^{0}\left(\mathbb{T}^{n}\right)$, so we also have injectivity there. The proof of Proposition 2.2 is complete, modulo the proof of Proposition 4.1, which we will give in $\S 4$.

The operators in (2.19)-(2.20) have inverses:

$$
\begin{aligned}
& E: B_{p, q}^{0}\left(\mathbb{T}^{n}\right) \longrightarrow B_{p, q}^{k}\left(\mathbb{T}^{n}\right), \\
& E: B_{p, q}^{-k}\left(\mathbb{T}^{n}\right) \longrightarrow B_{p, q}^{0}\left(\mathbb{T}^{n}\right) .
\end{aligned}
$$


It is readily verified that these maps coincide on elements in their common domains. Hence we can apply interpolation, to get:

Proposition 2.3 The operator $L$ has an inverse E, satisfying

$$
E: B_{p, q}^{s}\left(\mathbb{T}^{n}\right) \longrightarrow B_{p, q}^{s+k}\left(\mathbb{T}^{n}\right), \quad-k \leq s \leq 0 .
$$

REMARK. Here is an alternative proof of (2.22), valid for

$$
1<p<\infty, \quad-k<s<0 .
$$

Namely, parallel to (2.21), we have, from (2.18),

$$
\begin{aligned}
E: L^{p}\left(\mathbb{T}^{n}\right) & \longrightarrow H^{k, p}\left(\mathbb{T}^{n}\right), \\
E: H^{-k, p}\left(\mathbb{T}^{n}\right) & \longrightarrow L^{p}\left(\mathbb{T}^{n}\right),
\end{aligned}
$$

coinciding on elements in their common domain. We also have

$$
\left(H^{s_{0}, p}, H^{s_{1}, p}\right)_{\theta, q}=B_{p, q}^{s(\theta)},
$$

valid for

$$
s_{0} \neq s_{1}, \quad p \in(1, \infty), \quad q \in[1, \infty], \quad \theta \in(0,1), \quad s(\theta)=(1-\theta) s_{0}+\theta s_{1} .
$$

See [2], p. 153. Thus (2.22) follows via this interpolation from (2.24), as long as (2.23) holds. To be sure, it is the endpoint cases, not covered by (2.23), that are of greatest interest to us here.

\section{Local regularity, I}

In this section we use the results of $\S 2$ to prove Theorem 1.3. As noted in $\S 1$, we are reduced to proving (1.39), with $L_{2}$ replaced by $L_{1}$, which in turn can clearly be replaced by $L$, given by (2.17). The following accomplishes most of the remaining work.

Proposition 3.1 Let $P$ be as in Theorem 1.3, and let $L$ be derived from $P$ as discussed above. Given $\Omega \subset \mathbb{T}^{n}$ as in (1.39), $1 \leq j \leq k$, and $p, q \in[1, \infty]$,

$$
u \in B_{p, q(\mathrm{loc})}^{0}(\Omega), L u \in B_{p, q(\mathrm{loc})}^{-k+j}(\Omega) \Longrightarrow u \in B_{p, q(\mathrm{loc})}^{j}(\Omega) .
$$

The key to proving this is to combine (2.22) with the following commutator estimates. 
Lemma 3.2 Given $\mathcal{U} \subset \subset \Omega$ and $\rho \in C_{0}^{\infty}(\mathcal{U})$, we have

$$
[L, \rho]: B_{p, q}^{\sigma}(\mathcal{U}) \longrightarrow B_{p, q}^{\sigma-(k-1)}\left(\mathbb{T}^{n}\right), \quad 0 \leq \sigma \leq k-1 .
$$

Proof. From (1.37) we have that $L$ coincides with a differential operator on $\Omega$. Hence

$$
[L, \rho] u=\sum_{|\alpha| \leq k} b_{\alpha}(x)\left[\partial^{\alpha}, \rho\right] u, \quad b_{\alpha} \in C^{(\lambda)},
$$

which gives (3.2) for $\sigma=k-1$. (The fact that $B_{p, q}^{0}$ is a $C^{(\lambda)}$-module is a special case of Proposition B.1.) Since $L=L^{t}$, we also have

$$
[L, \rho] u=\sum_{|\alpha| \leq k}\left[(-\partial)^{\alpha}, \rho\right]\left(b_{\alpha}^{*} u\right),
$$

which gives (3.2) for $\sigma=0$. The rest follows by interpolation.

To begin the proof of Proposition 3.1, assume $u \in B_{p, q \text { (loc) }}^{0}(\Omega), L u \in B_{p, q \text { (loc })}^{-k+j}(\Omega)$ and pick $\rho \in C_{0}^{\infty}(\Omega)$. Pick $\mathcal{U} \subset \subset \Omega$ such that $\rho \in C_{0}^{\infty}(\mathcal{U})$. We have

$$
L(\rho u)=\rho L u+[L, \rho] u,
$$

with

$$
\rho L u \in B_{p, q}^{-k+j}\left(\mathbb{T}^{n}\right), \quad[L, \rho] u \in B_{p, q}^{-k+1}\left(\mathbb{T}^{n}\right),
$$

and hence

$$
\rho u=E(\rho L u+[L, \rho] u) \in B_{p, q}^{1}\left(\mathbb{T}^{n}\right) .
$$

This allows us to replace the hypothesis in (3.1) by

$$
u \in B_{p, q(\mathrm{loc})}^{1}(\Omega), \quad L u \in B_{p, q(\mathrm{loc})}^{-k+j}(\Omega) .
$$

Now if $k \geq 2$ and $j \geq 2$, we see that (3.5) holds with

$$
\rho L u \in B_{p, q}^{-k+j}\left(\mathbb{T}^{n}\right), \quad[L, \rho] u \in B_{p, q}^{-k+2}\left(\mathbb{T}^{n}\right),
$$

and hence

$$
\rho u=E(\rho L u+[L, \rho] u) \in B_{p, q}^{2}\left(\mathbb{T}^{n}\right) .
$$

Thus we can say $u \in B_{p, q \text { (loc) }}^{2}(\Omega)$. Iterating this argument (if $k>2$ and $j>2$ ) we arrive at a proof of (3.1).

We almost have Theorem 1.3, which is as in Proposition 3.1 but with (3.1) extended to

$$
u \in B_{p_{1}, q_{1}(\mathrm{loc})}^{0}(\mathcal{O}), P u \in B_{p, q(\mathrm{loc})}^{-k+j}(\mathcal{O}) \Longrightarrow u \in B_{p, q(\mathrm{loc})}^{j}(\mathcal{O}) .
$$


In the worst case, one has $p_{1}=1, q_{1}=\infty$. Then one pass through Proposition 3.1 gives

$$
u \in B_{1, \infty \text { (loc) }}^{j}(\mathcal{O}) \subset B_{p_{2}, q_{2}(\mathrm{loc})}^{0}(\mathcal{O}),
$$

with $p_{2}>1, q_{2}<\infty$. If $B_{p_{2}, q_{2}}^{0} \subset B_{p, q}^{0}$ we are done. Otherwise, repeated applications of Proposition 3.1 yield $u \in B_{p_{\nu}, q_{\nu}(\text { loc })}^{0}(\mathcal{O})$ and after a finite number of iterations one obtains $u \in B_{p, q(\text { loc })}^{0}(\mathcal{O})$; then one final iteration of Proposition 3.1 finishes the proof of (3.11).

\section{Local regularity, II}

In this section we establish two local regularity results that were used in $\S \S 1-2$. The first result completes the proof of Proposition 2.2.

Proposition 4.1 Assume $P$ is an elliptic differential operator of order $k$ on $\mathcal{O}$, and that

$$
P^{t} \text { has coefficients in } C^{(\lambda)} \text {, }
$$

with

$$
\lambda(j)=\langle j\rangle^{-s}, \quad s>1 .
$$

Then

$$
u \in B_{1, \infty \text { (loc) }}^{0}(\mathcal{O}), P u \in B_{1,1 \text { (loc) }}^{-k}(\mathcal{O}) \Longrightarrow u \in B_{1,1 \text { (loc) }}^{0}(\mathcal{O}) .
$$

Proof. The hypothesis says

$$
P u=\sum_{|\alpha| \leq k} \partial^{\alpha}\left(a_{\alpha} u\right), \quad a_{\alpha} \in C^{(\lambda)} .
$$

We use symbol smoothing to write

$$
P=P^{\#}+P^{b},
$$

where $P^{\#} \in O P S_{1, \delta}^{k}$ is elliptic, with parametrix $E^{\#} \in O P S_{1, \delta}^{-k}$ and

$$
P^{b}=\sum_{|\alpha| \leq k} \partial^{\alpha} A_{\alpha}^{b}, \quad A_{\alpha}^{b} \in O P C^{(\lambda)} S_{1,(\tau)}^{-\psi}
$$

Here $\psi$ and $\tau$ are as in (2.8), with $0<\gamma<s-1$. We have

$$
u=E^{\#} P u-\sum_{|\alpha| \leq k} E^{\#} \partial^{\alpha} A_{\alpha}^{b} u, \bmod C^{\infty} .
$$

Note that

$$
P u \in B_{1,1}^{-k} \Longrightarrow E^{\#} P u \in B_{1,1}^{0} \text {. }
$$


(For notational simplicity we drop the "loc" notation.) We claim

$$
A_{\alpha}^{b} \in O P C^{(\lambda)} S_{1,(\tau)}^{-\psi} \Longrightarrow A_{\alpha}^{b}: B_{1, \infty}^{0} \rightarrow B_{1, p_{1}}^{0},
$$

as long as

$$
p_{1}(s-1-\gamma)>1 \text {, }
$$

and more generally

$$
A_{\alpha}^{b} \in O P C^{(\lambda)} S_{1,(\tau)}^{-\psi} \Longrightarrow A_{\alpha}^{b}: B_{1, p_{\nu}}^{0} \rightarrow B_{1, p_{\nu+1}}^{0},
$$

where we pick

$$
p_{\nu}=\frac{1+\varepsilon}{\nu(s-1-\gamma)}
$$

and adjust $\varepsilon \in(0,1)$ so that for some integer $\mu, p_{\mu}=1$. The mapping properties (4.11) hold for $\nu<\mu$. Given this, we see that

$$
\begin{aligned}
u \in B_{1, \infty}^{0},|\alpha| \leq k & \Longrightarrow E^{\#} \partial^{\alpha} A_{\alpha}^{b} u \in B_{1, p_{1}}^{0} \\
& \Longrightarrow u \in B_{1, p_{1}}^{0},
\end{aligned}
$$

and then that, for $\nu<\mu$,

$$
\begin{aligned}
u \in B_{1, p_{\nu}}^{0},|\alpha| \leq k & \Longrightarrow E^{\#} \partial^{\alpha} A_{\alpha}^{b} u \in B_{1, p_{\nu+1}}^{0} \\
& \Longrightarrow u \in B_{1, p_{\nu+1}}^{0},
\end{aligned}
$$

which gives the desired conclusion (4.3) in Proposition 4.1.

Turning to the proof of (4.9) and (4.11), we have from Proposition B.1 that

$$
O P C^{(\lambda)} S_{1,(\tau)}^{0}: B_{p, q}^{0} \longrightarrow B_{p, q}^{0},
$$

under our hypotheses. To obtain (4.9)-(4.11) it hence suffices to note that

$$
q(D): B_{1, \infty}^{0} \longrightarrow B_{1, p_{1}}^{0}, \quad q(D): B_{1, p_{\nu}}^{0} \longrightarrow B_{1, p_{\nu+1}}^{0},
$$

when

$$
q(\xi)=\left(\log _{2}\langle\xi\rangle\right)^{-(s-1-\gamma)}, \quad s-1-\gamma>0,
$$

which can be arranged as long as $s>1$. This proves Proposition 4.1.

Finally, we prove Theorem 1.4, whose statement we recall: 
Proposition 4.2 Assume $P$ is an elliptic differential operator of order $k$ on $\mathcal{O}$, and that

$$
P \text { has coefficients in } C^{(\lambda)}, \quad \lambda(j)=\langle j\rangle^{-s}, \quad s>1,
$$

and that

$$
P \text { has Dini continuous coefficients. }
$$

Then

$$
u \in B_{\infty, \infty \text { (loc })}^{k}(\mathcal{O}), P u \in \operatorname{bmo}(\mathcal{O}) \Longrightarrow u \in \mathfrak{h}_{\text {loc }}^{k, \infty}(\mathcal{O}) .
$$

Proof. The hypothesis says

$$
P u=\sum_{|\alpha| \leq k} a_{\alpha} \partial^{\alpha} u, \quad a_{\alpha} \in C^{(\lambda)} .
$$

We use symbol smoothing to write $P=P^{\#}+P^{b}$, where $P^{\#} \in O P S_{1, \delta}^{k}$ is elliptic, with parametrix $E^{\#} \in O P S_{1, \delta}^{-k}$, and

$$
P^{b}=\sum_{|\alpha| \leq k} A_{\alpha}^{b} \partial^{\alpha}, \quad A_{\alpha}^{b} \in O P C^{(\lambda)} S_{1,(\tau)}^{-\psi} .
$$

Here $\psi$ and $\tau$ are as in (4.6), i.e., as in (2.8), with $0<\gamma<s-1$. We have

$$
u=E^{\#} P u-\sum_{|\alpha| \leq k} E^{\#} A_{\alpha}^{b} \partial^{\alpha} u, \bmod C^{\infty} .
$$

Of course

$$
P u \in \mathrm{bmo} \Longrightarrow E^{\#} P u \in \mathfrak{h}^{k, \infty},
$$

while

$$
u \in B_{\infty, \infty}^{k} \Longrightarrow \partial^{\alpha} u \in B_{\infty, \infty}^{0}, \quad|\alpha| \leq k
$$

We claim that

$$
A_{\alpha}^{b} \in O P C^{(\lambda)} S_{1,(\tau)}^{-\psi} \Longrightarrow A_{\alpha}^{b}: B_{\infty, \infty}^{0} \rightarrow B_{\infty, p_{1}}^{0}
$$

and more generally

$$
A_{\alpha}^{b} \in O P C^{(\lambda)} S_{1,(\tau)}^{-\psi} \Longrightarrow A_{\alpha}^{b}: B_{\infty, p_{\nu}}^{0} \rightarrow B_{\infty, p_{\nu+1}}^{0}
$$

with $p_{\nu}$ as in (4.12), so that $p_{\mu}=1$ for some integer $\mu$. The proof is the same as that of (4.9)-(4.11). Given this, it follows that

$$
\begin{aligned}
u \in B_{\infty, \infty}^{k},|\alpha| \leq k & \Longrightarrow E^{\#} A_{\alpha}^{b} \partial^{\alpha} u \in B_{\infty, p_{1}}^{k} \\
& \Longrightarrow u \in \mathfrak{h}^{k, \infty}+B_{\infty, p_{1}}^{k},
\end{aligned}
$$


the last line by (4.24). To continue, we iterate this argument, and use the fact that

$$
P^{b}=P-P^{\#}: \mathfrak{h}^{k, \infty} \longrightarrow \mathrm{bmo}
$$

itself a consequence of results in Chapter I, $\S 2$ of [13]. It is at this point that we make use of the assumption that the coefficients of $P$ are Dini continuous. To wit, the space bmo is a module over the algebra of Dini continuous functions, but results just cited require $s>2$ for bmo to be a module over $C^{(\lambda)}$. We conclude that, for $\nu<\mu$,

$$
\begin{aligned}
u \in \mathfrak{h}^{k, \infty}+B_{\infty, p_{\nu}}^{k} & \Longrightarrow E^{\#} P^{b} u \in \mathfrak{h}^{k, \infty}+B_{\infty, p_{\nu+1}}^{k} \\
& \Longrightarrow u \in \mathfrak{h}^{k, \infty}+B_{\infty, p_{\nu+1}}^{k} .
\end{aligned}
$$

This gives the conclusion (4.20) in Proposition 4.2.

Remark. We can replace the hypothesis (4.19) in Proposition 4.2 by the hypothesis

$$
P \text { has coefficients in } C^{\sigma}, \quad \sigma(h)=\left(\log \frac{1}{h}\right)^{-1} \text { for } h<<1,
$$

since bmo is a module over such $C^{\sigma}$ (cf. [14], Proposition 3.1).

Acknowledgements Work supported by NSF grant DMS-0758320.

\section{A Rough pseudodifferential operators and their symbol classes}

A pseudodifferential operator $P=p(x, D)$ with symbol $p(x, \xi)$ is given by

$$
P u(x)=(2 \pi)^{-n} \iint p(x, \xi) e^{i(x-y) \cdot \xi} u(y) d y d \xi .
$$

There are various symbol classes of interest, such as

$$
\begin{gathered}
S_{1,0}^{m}, \quad S_{\mathrm{cl}}^{m}, \quad S_{1, \delta}^{m}, \\
C^{(\lambda)} S_{1,0}^{m}, \quad C^{(\lambda)} S_{\mathrm{cl}}^{m}, \\
C^{(\lambda)} S_{1,(\tau)}^{m}, \quad C^{(\lambda)} S_{1,(\tau)}^{-\psi},
\end{gathered}
$$

which we will define below. If $\mathcal{S}$ is such a symbol class, and $p(x, \xi) \in \mathcal{S}$, we say $P$, defined by (A.1), belongs to $O P \mathcal{S}$. Thus we have the operator classes $O P S_{1,0}^{m}, O P C^{(\lambda)} S_{\mathrm{cl}}^{m}$, etc. If $p(x, \xi) \in \mathcal{S}$, the operator $\widetilde{P}$, given by

$$
\widetilde{P} u(x)=(2 \pi)^{-n} \iint p(y, \xi) e^{i(x-y) \cdot \xi} u(y) d y d \xi,
$$


is said to belong to $\varnothing P \mathcal{S}$. Adjoints of operators of the form (A.1) have the form (A.3).

The first three symbol classes listed in (A.2) are classes of smooth symbols. By definition, given $m \in \mathbb{R}$, one has

$$
p(x, \xi) \in S_{1,0}^{m} \Leftrightarrow\left|D_{x}^{\beta} D_{\xi}^{\alpha} p(x, \xi)\right| \leq C_{\alpha \beta}\langle\xi\rangle^{m-|\alpha|} .
$$

Here and below we set $\langle\xi\rangle=\left(2+|\xi|^{2}\right)^{1 / 2}$. We say $p(x, \xi) \in S_{\mathrm{cl}}^{m}$ provided $p(x, \xi) \in S_{1,0}^{m}$ and there is an asymptotic expansion

$$
p(x, \xi) \sim \sum_{j \geq 0} p_{m-j}(x, \xi),
$$

where $p_{m-j}(x, \xi) \in S_{1,0}^{m-j}$ and, for $|\xi| \geq 1, r \geq 1, p_{m-j}(x, r \xi)=r^{m-j} p_{m-j}(x, \xi)$. The meaning of (A.5) is that the difference between $p(x, \xi)$ and the sum over $j<k$ belongs to $S_{1,0}^{m-k}$. Next, given $\delta \in(0,1)$,

$$
p(x, \xi) \in S_{1, \delta}^{m} \Leftrightarrow\left|D_{x}^{\beta} D_{\xi}^{\alpha} p(x, \xi)\right| \leq C_{\alpha \beta}\langle\xi\rangle^{m-|\alpha|+\delta|\beta|} .
$$

We have $S_{\mathrm{cl}}^{m} \subset S_{1,0}^{m} \subset S_{1, \delta}^{m}$. Operators with such symbols have good mapping properties on $L^{p}$-Sobolev spaces, for $1<p<\infty$, on bmo-Sobolev spaces, on various Besov spaces, etc. A more detailed discussion can be found in Chapter I of [13].

The rest of the symbol classes listed in (A.2) are classes of rough symbols. By definition,

$$
\begin{aligned}
p(x, \xi) \in C^{(\lambda)} S_{1,0}^{m} \Leftrightarrow & \left|D_{\xi}^{\alpha} p(x, \xi)\right| \leq A_{\alpha}\langle\xi\rangle^{m-|\alpha|}, \text { and } \\
& \left\|D_{\xi}^{\alpha} p(\cdot, \xi)\right\|_{C^{(\lambda)}} \leq A_{\alpha}\langle\xi\rangle^{m-|\alpha|} .
\end{aligned}
$$

Here, $C^{(\lambda)}$ is the function space defined by (1.21). If $\lambda(j)=\langle j\rangle^{-s}$ and $s>1$, the first condition in (A.7) is redundant. We say $p(x, \xi) \in C^{(\lambda)} S_{\mathrm{cl}}^{m}$ if $p(x, \xi) \in C^{(\lambda)} S_{1,0}^{m}$ and there is an asymptotic expansion like (A.5). For example, a differential operator $P$ of order $m$, with coefficients in $C^{(\lambda)}$, would have symbol in $C^{(\lambda)} S_{\mathrm{cl}}^{m}$. We mention that, if instead of (1.22), we took $\lambda(j)=2^{-j r}$, then $C^{(\lambda)}$ is the space $C^{r}$ of Hölder continuous functions of exponent $r$ if $r \in(0,1)$, and it is the Zygmund space $C_{*}^{r}$ for general $r>0$. The study of operators in $O P C_{*}^{r} S_{1,0}^{m}$ is useful in the study of partial differential equations with Hölder continuous coefficients. The operator classes $O P C^{(\lambda)} S_{1,0}^{m}$ are similarly useful in the study of partial differential equations whose coefficients have weaker moduli of continuity.

The last two symbol classes listed in (A.2) arise from the previous two via the process of "symbol smoothing," which we will discuss below. First, we define these symbol classes. As before, $m \in \mathbb{R}$, and now $\tau$ and $\psi$ are functions of $\xi$. The particular cases of interest to us here are

$$
\langle\xi\rangle^{-\psi(\xi)}=\left(\log _{2}\langle\xi\rangle\right)^{-(s-1-\gamma)}, \quad \tau(\xi)=\left(\log _{2}\langle\xi\rangle\right)^{s-1-\gamma}
$$


with $s>1, \gamma \in(0, s-1)$, and, as in (1.22),

$$
\lambda(j)=\langle j\rangle^{-s} .
$$

We say

$$
\begin{aligned}
p(x, \xi) \in C^{(\lambda)} S_{1,(\tau)}^{m} \Leftrightarrow & \left|D_{\xi}^{\alpha} p(x, \xi)\right| \leq A_{\alpha}\langle\xi\rangle^{m-|\alpha|}, \text { and } \\
& \left\|D_{\xi}^{\alpha} p(\cdot, \xi)\right\|_{C^{(\lambda)}} \leq A_{\alpha} \tau(\xi)\langle\xi\rangle^{m-|\alpha|} .
\end{aligned}
$$

Furthermore, we say

$$
\begin{aligned}
p(x, \xi) \in C^{(\lambda)} S_{1,(\tau)}^{-\psi} \Leftrightarrow & \left|D_{\xi}^{\alpha} p(x, \xi)\right| \leq A_{\alpha}\langle\xi\rangle^{-\psi(\xi)-|\alpha|}, \text { and } \\
& \left\|D_{\xi}^{\alpha} p(\cdot, \xi)\right\|_{C^{(\lambda)}} \leq A_{\alpha} \tau(\xi)\langle\xi\rangle^{-\psi(\xi)-|\alpha|} .
\end{aligned}
$$

Operators whose symbols have such limited smoothness act on a correspondingly smaller array of function spaces than those with symbols in $S_{1, \delta}^{m}$, but there exist a number of useful results, given in Chapter I of [13], and supplemented by Appendix B here.

We finally say something about symbol smoothing, which writes

$$
p(x, \xi)=p^{\#}(x, \xi)+p^{b}(x, \xi) .
$$

For this, we choose $\delta \in(0,1)$ and set

$$
p^{\#}(x, \xi)=\sum_{j \geq 0} \Psi_{0}\left(2^{-j \delta} D_{x}\right) p(x, \xi) \psi_{j}(\xi),
$$

where $\left\{\psi_{j}\right\}$ is a Littlewood-Paley partition of unity, such as used in (1.21), and we pick $\Psi_{0}(\xi) \in C_{0}^{\infty}\left(\mathbb{R}^{n}\right)$, equal to 1 for $|\xi| \leq 1$. In (A.12), $p^{\#}(x, \xi)$ is the smoothed symbol and $p^{b}(x, \xi)$ is the remainder. Of use in the study of partial differential equations with Hölder continuous coefficients is the fact that, for such a decomposition, if $r>0$,

$$
\begin{aligned}
p(x, \xi) \in C_{*}^{r} S_{1,0}^{m} \Rightarrow p^{\#}(x, \xi) & \in S_{1, \delta}^{m}, \text { and } \\
p^{b}(x, \xi) & \in C_{*}^{r} S_{1, \delta}^{m-r \delta},
\end{aligned}
$$

where (somewhat parallel to (A.11))

$$
\begin{aligned}
q(x, \xi) \in C_{*}^{r} S_{1, \delta}^{\mu} \Leftrightarrow & \left|D_{\xi}^{\alpha} q(x, \xi)\right| \leq A_{\alpha}\langle\xi\rangle^{\mu-|\alpha|}, \text { and } \\
& \left\|D_{\xi}^{\alpha} q(\cdot, \xi)\right\|_{C_{*}^{r}} \leq A_{\alpha}\langle\xi\rangle^{\mu-|\alpha|+\delta r} .
\end{aligned}
$$

See [12], Chapter 13, Proposition 9.9. Of use in the current setting is that, if $\psi, \tau$, and $\lambda$ are given by (A.8)-(A.9), with $s>1, \gamma \in(0, s-1)$, then

$$
\begin{aligned}
p(x, \xi) \in C^{(\lambda)} S_{1,0}^{0} \Rightarrow p^{\#}(x, \xi) & \in S_{1, \delta}^{0}, \quad \text { and } \\
p^{b}(x, \xi) & \in C^{(\lambda)} S_{1,(\tau)}^{-\psi} .
\end{aligned}
$$

This is proven in [13], Chapter I, pp. 33-34. 


\section{B Rough pseudodifferential operators on $B_{p, q}^{0}$}

Here we prove the following result, which played an important role in $\S \S 2$ and 4.

Proposition B.1 Assume $P \in O P C^{(\lambda)} S_{1,(\tau)}^{0}$, with

$$
\lambda(j)=\langle j\rangle^{-s}, \quad \tau(\xi)=(\log \langle\xi\rangle)^{s-1-\gamma}, \quad s>1, \quad 0<\gamma<s-1 .
$$

Then

$$
P: B_{p, q}^{0} \longrightarrow B_{p, q}^{0}, \quad 1 \leq p, q \leq \infty .
$$

This was proven for $q=1$ in Proposition 12.2, Chapter I of [13]. We will recall that argument, modify it to treat the case $q=\infty$, and then obtain (B.2) by interpolation. As in [13] (following a strategy initiated in [3]) it suffices to treat $P=p(x, D)$ when $p(x, \xi)$ is an "elementary symbol"

$$
p(x, \xi)=\sum_{k=0}^{\infty} Q_{k}(x) \varphi_{k}(\xi)=\sum_{k, j=0}^{\infty} Q_{k j}(x) \varphi_{k}(\xi),
$$

where $\varphi_{k}(\xi)$ is smooth and supported on $\langle\xi\rangle \sim 2^{k}$, with $\varphi_{k}(\xi)=\varphi_{1}\left(2^{-k+1} \xi\right)$ for $k \geq 2$, and $Q_{k}$ satisfies

$$
\left\|Q_{k}\right\|_{L^{\infty}} \leq A_{1}, \quad\left\|Q_{k j}\right\|_{L^{\infty}} \leq C \lambda(j) \tau\left(2^{k}\right)
$$

Here $Q_{k j}=\psi_{j}(D) Q_{k}$, where $\left\{\psi_{j}\right\}$ is a Littlewood-Paley partition of unity. We form

$$
Q_{k}^{1}=\sum_{j \leq k-4} Q_{k j}, \quad Q_{k}^{2}=\sum_{j=k-3}^{k+3} Q_{k j}, \quad Q_{k}^{3}=\sum_{j \geq k+4} Q_{k j},
$$

and separately analyze

$$
p_{\mu}(x, D) f=\sum_{k=0}^{\infty} Q_{k}^{\mu}(x) \varphi_{k}(D) f, \quad \mu=1,2,3 .
$$

Recall that

$$
f \in B_{p, q}^{0}\left(\mathbb{R}^{n}\right) \Longleftrightarrow \sum_{\ell=0}^{\infty}\left\|\psi_{\ell}(D) f\right\|_{L^{p}}^{q}<\infty,
$$

if $1 \leq q<\infty$, and

$$
f \in B_{p, \infty}^{0}\left(\mathbb{R}^{n}\right) \Longleftrightarrow \sup _{\ell}\left\|\psi_{\ell}(D) f\right\|_{L^{p}}<\infty .
$$


To treat the $\mu=1$ case of (B.6), we use the fact that $Q_{k}^{1} \varphi_{k}(D) f$ has Fourier transform supported in $|\xi| \sim 2^{k}$ to write

$$
\psi_{\ell}(D) p_{1}(x, D) f=\sum_{k=\ell-5}^{\ell+5} \psi_{\ell}(D)\left(Q_{k}^{1} \varphi_{k}(D) f\right),
$$

hence, given $\left\|Q_{k}\right\|_{L^{\infty}} \leq A_{1}$,

$$
\left\|\psi_{\ell}(D) p_{1}(x, D) f\right\|_{L^{p}} \leq C A_{1} \sum_{k=\ell-5}^{\ell+5}\left\|\varphi_{\ell}(D) f\right\|_{L^{p}} .
$$

Hence

$$
\left\|Q_{k}\right\|_{L^{\infty}} \leq A_{1}<\infty \Longrightarrow p_{1}(x, D): B_{p, q}^{0}\left(\mathbb{R}^{n}\right) \rightarrow B_{p, q}^{0}\left(\mathbb{R}^{n}\right),
$$

for all $p, q \in[1, \infty]$.

To treat the $\mu=2$ case of (B.6), we know that $Q_{k}^{2} \varphi_{k}(D) f$ has Fourier transform supported in $|\xi| \leq C 2^{k}$, and we can write

$$
\psi_{\ell}(D) p_{2}(x, D) f=\sum_{k \geq \ell-4} \psi_{\ell}\left(Q_{k}^{2} \varphi_{k}(D) f\right) .
$$

Hence

$$
\left\|\psi_{\ell}(D) p_{2}(x, D) f\right\|_{L^{p}} \leq C \sum_{k \geq \ell-4}\left\|Q_{k}^{2}\right\|_{L^{\infty}}\left\|\varphi_{k}(D) f\right\|_{L^{p}}
$$

The second estimate in (B.4) gives

$$
\left\|Q_{k}^{2}\right\|_{L^{\infty}} \leq C \lambda(k) \tau\left(2^{k}\right) \leq C\langle k\rangle^{-1-\gamma},
$$

with $\gamma \in(0,1)$. As in (12.17) in Chapter I of [13], we have the estimate

$$
\begin{aligned}
\sum_{\ell}\left\|\psi_{\ell}(D) p_{2}(x, D) f\right\|_{L^{p}} & \leq C \sum_{\ell \geq 0} \sum_{k \geq \ell-4}\langle k\rangle^{-1-\gamma}\left\|\varphi_{k}(D) f\right\|_{L^{p}} \\
& \leq C \sum_{k \geq 0}(k+4)\langle k\rangle^{-1-\gamma}\left\|\varphi_{k}(D) f\right\|_{L^{p}}
\end{aligned}
$$

and hence

$$
p_{2}(x, D): B_{p, 1}^{0} \longrightarrow B_{p, 1}^{0}, \quad 1 \leq p \leq \infty .
$$

Furthermore, we have

$$
\begin{aligned}
\sup _{\ell \geq 0}\left\|\psi_{\ell}(D) p_{2}(x, D) f\right\|_{L^{p}} & \leq C \sup _{\ell \geq 0} \sum_{k \geq \ell-4}\langle k\rangle^{-1-\gamma}\left\|\varphi_{k}(D) f\right\|_{L^{p}} \\
& \leq C \sup _{k \geq 0}\left\|\varphi_{k}(D) f\right\|_{L^{p}}
\end{aligned}
$$


and hence

$$
p_{2}(x, D): B_{p, \infty}^{0} \longrightarrow B_{p, \infty}^{0}, \quad 1 \leq p \leq \infty .
$$

Now there is the interpolation result

$$
\left(B_{p, 1}^{0}, B_{p, \infty}^{0}\right)_{\theta, q}=B_{p, q}^{0}, \quad \frac{1}{q}=1-\theta,
$$

cf. [2], p. 153. Hence (B.16) and (B.18) imply

$$
p_{2}(x, D): B_{p, q}^{0} \longrightarrow B_{p, q}^{0}, \quad p, q \in[1, \infty] .
$$

Now we treat the $\mu=3$ case of (B.6). For $j \geq k+4, Q_{k j} \varphi_{k}(D) f$ has Fourier transform with support in $|\xi| \sim 2^{j}$. Hence

$$
\psi_{\ell}(D) p_{3}(x, D) f=\sum_{k \leq \ell+5} \sum_{j=\ell-5}^{\ell+5} \psi_{\ell}(D)\left(Q_{k j} \varphi_{k}(D) f\right),
$$

so

$$
\begin{aligned}
\left\|\psi_{\ell}(D) p_{3}(x, D) f\right\|_{L^{p}} & \leq C \sum_{k \leq \ell+5} \sum_{j=\ell-5}^{\ell+5}\left\|Q_{k j}\right\|_{L^{\infty}}\left\|\varphi_{k}(D) f\right\|_{L^{p}} \\
& \leq C \sum_{k \leq \ell+5} \lambda(\ell) \tau\left(2^{k}\right)\left\|\varphi_{k}(D) f\right\|_{L^{p}} .
\end{aligned}
$$

Summing over $\ell$, we interchange the order of $(\ell, k)$ summation and use

$$
\sum_{\ell \geq k-5} \lambda(\ell) \tau\left(2^{k}\right) \leq A_{2}<\infty,
$$

which follows from (B.1), to obtain

$$
\sum_{\ell \geq 0}\left\|\psi_{\ell}(D) p_{3}(x, D) f\right\|_{L^{p}} \leq C A_{2} \sum_{k=0}^{\infty}\left\|\varphi_{k}(D) f\right\|_{L^{p}},
$$

and hence

$$
p_{3}(x, D): B_{p, 1}^{0} \longrightarrow B_{p, 1}^{0}, \quad 1 \leq p \leq \infty .
$$

To handle $q=\infty$, we have

$$
\begin{aligned}
\sup _{\ell \geq 0}\left\|\psi_{\ell}(D) p_{3}(x, D) f\right\|_{L^{p}} & \leq C \sup _{\ell \geq 0} \sum_{k \leq \ell+5} \lambda(\ell) \tau\left(2^{k}\right)\left\|\varphi_{k}(D) f\right\|_{L^{p}} \\
& \leq C A_{3} \sup _{k \geq 0}\left\|\varphi_{k}(D) f\right\|_{L^{p}},
\end{aligned}
$$


with

$$
A_{3}=\sup _{\ell \geq 0} \sum_{k \leq \ell+5} \tau\left(2^{k}\right) \lambda(\ell)<\infty
$$

by (B.1). Hence

$$
p_{3}(x, D): B_{p, \infty}^{0} \longrightarrow B_{p, \infty}^{0}, \quad 1 \leq p \leq \infty,
$$

and another application of (B.19) yields

$$
p_{3}(x, D): B_{p, q}^{0} \longrightarrow B_{p, q}^{0}, \quad p, q \in[1, \infty] .
$$

This completes the proof of Proposition B.1.

\section{References}

[1] S. Agmon, A. Douglis, and L. Nirenberg, Estimates near the boundary for solutions of elliptic partial differential equations satisfying general boundary conditions, Comm. Pure Appl. Math. 12 (1959), 623-727.

[2] J. Bergh and J. Löfström, Interpolation Spaces, an Introduction, Springer-Verlag, New York, 1976.

[3] R. Coifman and Y. Meyer, Au dela des Opérateurs Pseudodifférentiels, Asterisque \#57, Soc. Math. de France, 1978.

[4] R. Coifman, R. Rochberg, and G. Weiss, Factorization theorems for Hardy spaces in several variables, Ann. Math. 103 (1976), 611-635.

[5] C. Fefferman and E. Stein, $H^{p}$ spaces of several variables, Acta Math. 129 (1972), $137-193$.

[6] D. Goldberg, A local version of real Hardy spaces, Duke Math. J. 46 (1979), 27-42.

[7] P. Hartman and A. Wintner, On the existence of Riemannian manifolds which cannot carry non-constant analytic or harmonic functions in the small, Amer. J. Math. 75 (1953), 260-276.

[8] P. Hartman and A. Wintner, On uniform Dini conditions in the theory of linear partial differential equations of elliptic type, Amer. J. Math. 77 (1955), 329-353.

[9] R. de la Llave, Remarks on Sobolev regularity in Anosov systems, Ergod. Theory and Dynam. Systems, 21 (2001), 1139-1180. 
[10] R. de la Llave, J. Marco, and R. Moriyon, Canonical perturbation theory of Anosov systems and regularity results for the Livsic cohomology equation, Annals of Math. 123 (1986), 537-611.

[11] J. Rauch and M. Taylor, Regularity of functions smooth along foliations, and elliptic regularity, J. Funct. Anal. 225 (2005), 74-93.

[12] M. Taylor, Partial Differential Equations, Vol. 3, Springer-Verlag, New York, 1996.

[13] M. Taylor, Tools for PDE, Math. Surv. and Monogr. \#81, American Math. Soc., Providence, R.I., 2000.

[14] M. Taylor, Hardy spaces and bmo on manifolds with bounded geometry, J. Geometric Analysis 19 (2009), 137-190.

[15] H. Triebel, Theory of Function Spaces, Birkhauser, Basel, 1983. 